secretion in metastatic neuroendocrine (NE) cancers. (2) To evaluate the expression pattern of SV2 receptors in NE cancer patient-derived tissues for prediction of patient response for recombinant HCR (rHCR) treatment. (3) To assess the in vivo efficacy and toxicity of $\mathrm{rHCR}$ in a NE cancer liver metastasis mouse models and in the NE patient-derived 3D MicroTumor system. (4) To collect preclinical data to design and conduct a clinical trial with NE cancer patients, a major goal toward translating our discoveries into much needed therapies. METHODS/STUDY POPULATION: Recombinant botulinum heavy chain ( $\mathrm{rHCR}$ ) was produced using an IPTG-inducible expression vector in $E$. coli BL2I. The rHCR was His-Tag purified and stored in PBS buffer before usage. Cytotoxicity: H727, TT, and MZ cells were plated at a density of 5000 cells/well in 96-well plates and incubated under standard conditions overnight. The next day, cells were treated with 10,100 , or $500 \mathrm{nmol} / \mathrm{L}$ of $\mathrm{rHCR}$ and incubated for 72 hours. Following incubation, cell viability was assessed by ATP quantification using the CellTiter-Glo (Promega) assay. Fresh NE tumors were dissociated and injected into polydimethylsiloxane bioreactors in a matrigel and collagen suspension for 3D culture experiments. The viability of 3D cultures incubated with various doses of $r \mathrm{HCR}$ was assessed by measuring the uptake of the near-infrared dye IR-783 using an IVIS imaging system. Western blot: $\mathrm{H} 727, \mathrm{TT}$, and MZ cells were seeded in 6-well plates at a density of $3 \times 105$ cells/well for 24 hours followed by treatment with $100 \mathrm{nmol} / \mathrm{L}$ for 72 hours. Total cellular proteins were isolated and analyzed to assess the level of SV2A expression and the effect of rHCR on the expression levels of NET marker proteins. Immunohistochemistry: Deparaffinized tissue culture slides were incubated with SV2A primary antibody in I\% BSA and incubated overnight at $4^{\circ} \mathrm{C}$. Slides were rinsed twice with TBS containing $0.025 \%$ Triton, followed by $0.3 \% \mathrm{H}_{2} \mathrm{O}_{2}$ for 15 minutes. Slides were then incubated with HRP-conjugated secondary antibody for I hour at room temperature. Detection of protein-protein interaction: Precleared cell lysate was incubated with glutathioneagarose beads in the presence of $10 \mu \mathrm{g}$ of GST-tagged rHCR for $2 \mathrm{~h}$ at $4^{\circ} \mathrm{C}$ with end-over end mixing. Samples were then centrifuged at $10,000 \mathrm{~g}$ for 2 minutes and the supernatant was analyzed by SDS-PAGE. Preclinical models: To allow rHCR testing on NET patient derived cells in a very novel 3D surrogates, sterile collected fresh NET tissues will be obtained from the UAB Tissue Procurement, dissociated into a single cell suspension and injected into a polydimethylsiloxane bioreactors containing extracellular matrix composed of bovine collagen and matrigel. Such 3D cell culture will be maintained in bioreactors with constant supply of media through the microchannels and treated with $\mathrm{rHCR}$ at concentrations ranging from $10 \mathrm{nM}$ to $1 \mathrm{mM}$. Following histologic confirmation of growth and morphology of NET patient-derived 3D surrogates we will test the anticancer activity of $\mathrm{rHCR}$ in this system using the standard cytotoxicity assays as well as we will validate the NET hormone expression using immunohistochemistry assay. To create an animal model of NE cancer progression, we will perform intrasplenic injection of NET cell lines. In approximately 4 weeks, the animals should develop NE liver metastases based upon our previous experience. rHCR-iFPs accumulation in the tumor mass: rHCR-iFPs will be injected to the tumor bearing mice after 4 weeks of cells implantation in I week interval for total of 4 treatments at the concentrations of $0.125,1.25$, and $12.5 \mathrm{mg} / \mathrm{kg}$. RESULTS/ANTICIPATED RESULTS: Based on the preliminary data, we expect to detect rHCR-iFPs in NE cancer xenografts and in patient derived 3D explants. Our preliminary data revealed that treating NETcells with rHCR significantly reduced NE peptide expression in 3 days. Thus, we expect to see the decrease of NE tumor markers even if the fluorescent detection method is not sensitive enough to monitor the signal. The reduction of the NET markers can be used as an indicator of the rHCR-iFPs uptake by the tumor mass. If HCR exhibit high binding affinity to SV2 receptors in NET models but moderate anticancer efficacy, we plan to use rHCR peptide to conjugate with the nanocarrier for targeted drug delivery. In this case rHCR peptide can be used as a ligand that specifically binds to NE cancer cells and delivers anticancer drug. In 3D NET patient derived explants we expect significant reduction of NET markers and hormones (serotonin and calcitonin) in NE cancer cells upon long-term rHCR-iFPs treatment. In addition, we will perform multiplex protein quantification assay using Luminex to assess the various hormones, cytokines, and growth factors to be repurposed into a diagnostic and detection reagent, or a drug delivery ligand for targeted therapies. DISCUSSION/SIGNIFICANCE OF IMPACT: NE cancers are highly metastatic: NE cancers such as carcinoid, islet cell tumors, and medullary thyroid cancer frequently metastasize to the liver. They are the second most prevalent Gl malignancy. Ninety percent of patients with pancreatic carcinoid tumors and $50 \%$ of patients with islet cell tumors develop isolated hepatic metastases. Patients with untreated, isolated NE liver metastases have $<30 \% 5$-year survival. Thus, there is a critical need for new therapies for NE cancers. Surgery is the only curative therapy available for patients with NE cancers but most cannot be cured: surgical removal is the most effective treatment for NE cancers; however, a very high percentage of patients present with metastatic disease. While surgical resection can be potentially curative, many patients are not candidates for operative intervention due to widespread metastases or the degree of hepatic involvement by the NE cancers. Moreover, other forms of therapy including chemoembolization, radioembolization, cryoablation, and chemotherapy have had limited efficacy. We hope that our in vitro data on rHCR toxicity and specificity to NET, validated in the pre-clinical models will allow the first in-human application of this technology in clinical studies.
Targeted eccentric motor control to improve locomotion after incomplete spinal cord injury Kevin O'Brien, D. Michele Basso and James Schmiedeler Indiana University School of Medicine

OBJECTIVES/SPECIFIC AIMS: Incomplete spinal cord injury typically results in life-long disability, often in the form of profound loss of locomotion capability. Individuals who have experienced incomplete spinal cord injury exhibit persistent eccentric motor deficits, which are particularly prevalent in the weight acceptance phase of gait and emphasized in sagittal plane knee motion and frontal plane hip motion. METHODS/STUDY POPULATION: Motion analysis can capture the kinematic and joint-level deficits of these individuals, but it is impossible to directly calculate the contributions of individual muscles to weight acceptance due to the complexity of the musculoskeletal system. Instead, those muscle contributions must be simulated in order to approximate muscle power during locomotion. RESULTS/ANTICIPATED RESULTS: The traditional method for driving these simulations with electromyography readings is unavailable for individuals who have neuromuscular deficits (e.g., spasticity or paralysis), due to the need to generate reliable maximum voluntary isometric contractions for baseline purposes. Instead, this research develops a novel method for using resting electromyography data to drive musculoskeletal simulations using a muscle activation threshold paradigm. DISCUSSION/SIGNIFICANCE OF IMPACT: The simulation results of this method more closely resemble experimental results, but further simulation refinement is needed to fully capture the true muscle activity.

\section{Targeting immunosuppressive myeloid cells to enhance cancer immunotherapy}

Xin Lu

Indiana University School of Medicine

OBJECTIVES/SPECIFIC AIMS: Prostate cancer (PCa) is the most common noncutaneous malignancy in men in the United States. A significant fraction of advanced $\mathrm{PCa}$ treated with androgen deprivation therapy experience relentless progression to lethal metastatic castration-resistant prostate cancer (mCRPC). The $\mathrm{PCa}$ tumor microenvironment is comprised of a complex mixture of epithelial and stroma cell types engaged in multifaceted heterotypic interactions functioning to maintain tumor growth and immune evasion. We recently uncovered the important role played by myeloid-derived suppressor cells (MDSCs) to mediate tumor immune evasion in aggressive PCa (Wang, Lu et al., Cancer Discovery, 2016). Immune checkpoint blockade (ICB) has elicited durable therapeutic responses across a number of cancer types. However, the impact of ICB on $m C R P C$ has been disappointing, which may signal the need to combine mechanistically-distinct ICB agents and/or override immunosuppression in the tumor microenvironment. Our objective is to determine if robust immunotherapy responses in mCRPC may be elicited by the combined actions of ICB agents together with targeted agents that neutralize MDSCs yet preserve $T$ cell function. METHODS/STUDY POPULATION: We created a novel embryonic stem cellbased chimeric mouse model of $\mathrm{mCRPC}$ engineered with signature mutations to study the response to single and combination immunotherapy. The efficacy studies were followed with detailed mechanistic investigation and clinical validation RESULTS/ANTICIPATED RESULTS: Consonant with early stage clinical trials experience, anti-CTLA4 or anti-PDI monotherapy failed to impact disease progression. Similarly, modest anti-tumor activity was observed with combination ICB as well as monotherapy with targeted agents including Cabozantinib (tyrosine kinase inhibitor), Dactolisib (PI3K/mTOR inhibitor), and Dasatinib (tyrosine kinase inhibitor). In contrast, mCRPC primary and metastatic disease showed robust responses to dual ICB treatment together with either Cabozantinib or Dactolisib, but not with Dasatinib which impaired T cell infiltration in the tumor. Detailed intratumoral immune profiling with mass cytometry (CyTOF) showed that combined ICB and Cabozantinib or Dactolisib was associated with significant depletion of MDSCs. Cabozantinib and Dactolisib blocked the PI3K signaling activity in MDSCs and reduced their immunosupppresive activity. Mechanistically, the combination efficacy was due to the upregulation of IL-IRA and suppression of MDSC-promoting cytokines secreted by mCRPC cells. DISCUSSION/SIGNIFICANCE OF IMPACT: We demonstrated that an antibody cocktail targeting CTLA4 and PDI was insufficient to generate effective anti-tumor response, but combination of ICB with targeted therapy that inactivates PI3K signaling displayed superior synergistic efficacy through impairing MDSCs in the tumor microenvironment. These observations illuminate a clinical path hypothesis for combining ICB with MDSC-targeted therapies in the treatment of mCRPC. Importantly, conclusions from the study on PCa may have implications in combination immunotherapy for aggressive breast cancer which is also largely resistant to immune checkpoint blockade and replete with immunosuppressive myeloid cells. 